\title{
CARACTERIZAÇÃO DAS VÍTIMAS DE INFARTO DO MIOCÁRDIO ADMITIDAS EM UMA UNIDADE CORONARIANA
}

\section{CHARACTERIZATION OF THE VICTIMS OF MYOCARDIAL INFARCTION ADMITTED TO A CORONARY UNIT}

\section{CARACTERIZACIÓN DE LAS VÍCTIMAS DE INFARTO DEL MIOCARDIO ADMITIDAS EN UNA UNIDAD CORONARIA}

\author{
Danielle Santana Soares ${ }^{1}$, Thalita Silva Santos ${ }^{2}$, Suellen Rodrigues de Oliveira Maier ${ }^{3}$, \\ Mayara Rocha Siqueira Sudré ${ }^{4}$, Cezar Augusto da Silva Flores ${ }^{5}$, Wanmar de Souza Oliveira ${ }^{6}$
}

\begin{abstract}
RESUMO
Objetivo: caracterizar as vítimas de infarto agudo do miocárdio admitidas em uma unidade de terapia intensiva coronariana de um hospital no sul do estado de Mato Grosso. Metodologia: estudo transversal com amostra composta por 213 pacientes admitidos na unidade coronariana de um hospital no sul de Mato Grosso. Os dados foram coletados, por meio dos registros na unidade, de janeiro a dezembro do ano de 2017. A análise dos dados foi realizada por meio do programa Epi Info 3.5.1. Resultados: foram 67,6\% são do sexo masculino, 31,9\% apresentaram idade entre 65 e 74 anos, sendo 58,2\% provenientes dos serviços de urgência e emergência. O tempo de permanência foi de até 72 horas com $63,8 \%$ e o desfecho clínico foi 89,7\% de alta hospitalar. Conclusão: o conhecimento acerca das características dos pacientes admitidos na referida unidade pode favorecer o planejamento da assistência à saúde.
\end{abstract}

Descritores: Infarto do Miocárdio, Unidades de Terapia Intensiva, Perfil de Saúde.

\begin{abstract}
Objective: To characterize the victims of acute myocardial infarction admitted to a coronary intensive care unit of a hospital in the southern state of Mato Grosso. Methodology: crosssectional study with a sample of 213 patients admitted to the coronary unit of a hospital in southern Mato Grosso. Data were collected through records at the unit from January to December 2017. Data analysis was performed using the Epi Info 3.5.1 program. Results: 67.6\% were male, 31.9\% were aged between 65 and 74 years, and 58.2\% came from emergency services. The length of stay was up to 72 hours with $63.8 \%$ and the clinical outcome was $89.7 \%$ of hospital discharge. Conclusion: knowledge about the characteristics of patients admitted to this unit may favor health care planning.

Descriptors: Myocardial Infarction, Intensive Care Units, Health Profile.

\footnotetext{
${ }^{1}$ Graduada de Enfermagem, Universidade Federal de Mato Grosso, Campus Universitário de Rondonópolis.

${ }^{2}$ Graduada de Enfermagem, Universidade Federal de Mato Grosso.

${ }^{3}$ Enfermeira. Mestre em Educação. Professora Adjunta II da Universidade Federal de Mato Grosso, Campus Universitário de Rondonópolis.

${ }^{4}$ Enfermeira. Mestre em Enfermagem. Professora Assistente II da Universidade Federal de Mato Grosso, Campus Universitário de Rondonópolis.

${ }^{5}$ Enfermeiro e Pedagogo. Mestre em Educação. Professor Adjunto II da Universidade Federal de Mato Grosso, Campus Universitário de Sinop.

${ }^{6}$ Graduado de Enfermagem, Universidade Federal de Mato Grosso.
} 


\section{RESUMEN}

Objetivo: caracterizar a las víctimas de infarto agudo de miocardio ingresadas en una unidad de cuidados intensivos coronarios de un hospital en el estado sureño de Mato Grosso. Metodología: estudio transversal con una muestra de 213 pacientes ingresados en la unidad coronaria de un hospital en el sur de Mato Grosso. Los datos se recopilaron a través de los registros en la unidad de enero a diciembre de 2017. El análisis de los datos se realizó mediante el programa Epi Info 3.5.1. Resultados: $67.6 \%$ eran hombres, $31.9 \%$ tenían entre 65 y 74 años y $58.2 \%$ provenían de servicios de emergencia. La duración de la estancia fue de hasta 72 horas con un $63,8 \%$ y el resultado clínico fue del $89,7 \%$ del alta hospitalaria. Conclusión: el conocimiento sobre las características de los pacientes ingresados en esta unidad puede favorecer la planificación de la atención médica.

Descriptores: Infarto del Miocardio, Unidades de Cuidados Intensivos, Perfil de Salud.

\section{INTRODUÇÃO}

A Doença Arterial Coronariana (DAC) constitui uma classe das doenças cardiovasculares, a qual é causada por depósitos de placas ateroscleróticas na parte interna das artérias coronárias, causando estreitamento das mesmas e levando a um fornecimento inadequado de sangue ao músculo cardíaco. ${ }^{1}$

A DAC, tem como suas formas de apresentação, a Síndrome Coronariana Aguda (SCA) que, dentre suas classificações, há o Infarto Agudo do Miocárdio (IAM), o qual é causado pela ruptura ou erosão da placa aterosclerótica, levando à diminuição ou ausência da perfusão ao tecido cardíaco, o que causa desequilíbrio entre o aporte e a demanda de oxigênio e resulta no comprometimento celular irreversível e necrose muscular cardíaca. $^{2-3}$

Em relação aos fatores de risco para o IAM, podem ser classificados baseandose no grau em que podem ou não ser modificáveis a partir de alterações no estilo de vida e no comportamento individual. Neste caso, são fatores de riscos modificáveis a hipertensão arterial, hiperglicemia, diabetes mellitus, hipercolesterolemia, tabagismo, obesidade e sedentarismo. Em contrapartida, são fatores de risco não modificáveis o histórico familiar de DAC, idade, sexo e raça. ${ }^{4}$

No que se refere as manifestações clínicas do IAM, estas se apresentam como uma dor precordial, que pode se estender para o membro superior esquerdo e para região mandibular, acompanhado de sudorese, náusea e vômitos. Essa condição, pode apresentar-se após o quadro de angina, tornando-se uma situação emergencial que requer tratamento especializado imediato, principalmente em Unidades de Terapia Intensiva (UTI). ${ }^{4}$

Sob essa perspectiva, a UTI é um setor hospitalar destinado a usuários em situação clínica grave ou de risco clínico ou 
cirúrgico, dos quais necessitam de cuidados intensivos multidisciplinares. Dentre suas classificações, destaca-se a Unidade de Terapia Intensiva Coronariana (UCO), a qual visa realizar atendimento às vítimas de doenças cardiovasculares graves com alto risco de complicações. ${ }^{5}$

Deste modo, as doenças do aparelho circulatório são as mais comuns ocorridas em atendimentos e admissões nas unidades de emergência e de terapia intensiva, além de ser considerada a principal causa de mortalidade no Brasil, visto que, em 2017, o IAM foi responsável por 112.406 internações, e em 2016, registrou-se cerca de 94.148 óbitos. $^{6-7}$

Diante dos fatos expostos, surge o anseio de apurar a seguinte questão: Quais as características sociodemográficas e clínicas das vítimas de infarto agudo do miocárdio admitidas em uma unidade intensiva coronariana?

Nesta perspectiva o conhecimento acerca das características dos usuários admitidos na unidade pode favorecer a implementação da assistência de forma individualizada e holística. Portanto, o objetivo deste estudo foi caracterizar as vítimas de infarto agudo do miocárdio admitidas em uma unidade de terapia intensiva coronariana de um hospital no sul do estado de Mato Grosso.

\section{MÉTODO}

Trata-se de um estudo descritivo, do tipo transversal com abordagem quantitativa. Para a sua realização, foram obedecidos os preceitos éticos e legais em pesquisa com seres humanos, consoante a resolução $\mathrm{n}^{\mathrm{o}} 466 / 2012$ do Conselho Nacional de Saúde $^{8}$. Desta maneira, essa pesquisa possui parecer ético favorável $\mathrm{n}^{\circ}$ 1.931.153 com a Certificação de Apresentação para Apreciação Ética (CAAE) no 62895316.8.0000.8088.

O estudo foi desenvolvido em uma UCO de um hospital no sul de Mato Grosso. Esta unidade é composta por nove leitos de internação para adultos, recebendo usuários tanto de convênio de saúde público quanto privado.

A população do estudo foi constituída por 213 usuários que foram admitidos na UCO tendo como diagnostico IAM. Os dados coletados foram de janeiro a dezembro de 2017, através do registro interno e prontuário eletrônico, no período de janeiro a dezembro do ano de 2017, sendo registrados, em um instrumento, elaborado pelos autores, contendo informações referentes a: sexo, idade, setor de procedência, tempo de permanência e desfecho clínico.

A análise dos dados foi realizada através do programa Epi Info 3.5.1, sendo feita dupla digitação para ajustes das inconsistências. As variáveis contínuas foram descritas com média, mínima e 
máxima e as variáveis categóricas foram descritas com números absolutos (n) e frequências relativas (\%).

\section{RESULTADOS}

Conforme os achados, em 2017, a unidade coronariana de um hospital do sul do Mato Grosso, admitiu um total de 593 usuários, sendo que destes, 213 foram vítimas de IAM. A tabela 1 mostra as características sociodemográficas e clínicas destas vítimas. 
Tabela 1 - Características sociodemográficas e clínicas das vítimas de infarto do miocárdio admitidas na unidade coronariana. Mato Grosso, Brasil, 2017

\begin{tabular}{lccccc}
\hline Variáveis & n & \% & Média & Mín. & Máx. \\
\hline Sexo & 144 & 67,6 & & & \\
Masculino & 69 & 32,4 & & & \\
Feminino & & & & & \\
Idade & 3 & 1,4 & & & \\
$20-29$ anos & 4 & 1,9 & & & \\
$29-38$ anos & 22 & 10,3 & & & \\
38 - -47 anos & 27 & 12,7 & & & \\
$47-56$ anos & 50 & 23,5 & 63,5 & 20 & \multirow{2}{*}{98 anos } \\
$56-65$ anos & 68 & 31,9 & anos & anos & \\
$65-74$ anos & 30 & 14,1 & & & \\
$74-83$ anos & 8 & 3,8 & & & \\
83 - 92 anos & 1 & 0,5 & & & \\
$92-98$ anos & & & & &
\end{tabular}

\begin{tabular}{lccccc} 
Setor de Procedência & 16 & 7,5 & & & \\
Enfermarias & 69 & 32,4 & & & \\
Centro Cirúrgico & 124 & 58,2 & & & \\
Serviços de Urgência e Emergência & 1 & 0,5 & & & \\
Ambulatórios & 3 & 1,4 & & & \\
Outros Hospitais & & & & & \\
Tempo de Permanência & 136 & 63,8 & 96 & $>24$ & 600 \\
Até 72 horas & 77 & 36,2 & horas & horas & horas \\
Acima de 72 horas & & & & & \\
Desfecho clínico & 191 & 89,7 & & & \\
Alta & 22 & 10,3 & & & \\
Óbito & & & & & \\
\hline
\end{tabular}

Fonte: dados dos autores.

Nota: $\vdash=$ Frequência de amplitude. Mín.= Mínima. Máx.= Máxima.

Nota-se que houve predomínio de usuários do sexo masculino $(67,6 \%)$, e quanto à idade houve preponderância da faixa entre 65 e 74 anos $(31,9 \%)$, com média de 63,5 anos, sendo considerado como extremos de idade mínima 20 anos e de máxima 98 anos. A variável idade foi separada por grupos etários a partir do cálculo da classe e amplitude da frequência.
Quanto ao setor de procedência, houve predominância de vítimas oriundas dos serviços de urgência e emergência (58,2\%), uma vez que esse serviço é responsável por ofertar atendimento rápido e resolutivo, além de ser a porta de entrada do serviço hospitalar.

Ainda em relação a procedência, considera-se as enfermarias como as 
unidades de internação hospitalar, incluindo também as transferências de uma outra unidade crítica do hospital. Os dados correspondentes ao centro cirúrgico foram agrupados com os dos serviços de hemodinâmica, visto que, se compreendeu, previamente, à internação na UCO, a realização de procedimentos invasivos, diagnóstico ou correção.

Os serviços de urgência e emergência, inclui convênios público e privado do próprio hospital em estudo e de outros hospitais do município. Já o setor de ambulatório refere-se ao de especialidades médicas privadas. Por fim os dados de outros hospitais, considera-se que são as transferências externas para a unidade em estudo, tendo em vista que o mesmo é referência em atendimento especializado para acometimentos cardíacos.

No que concerne a tempo de permanência na UCO, identificou-se que houve prevalência ao tempo de até 72 horas (63,8\%), apresentando média de aproximadamente 96 horas, variando entre o mínimo inferior à 24 horas e máximo de 600 horas. Utilizou-se como base de análise as 72 horas, tendo em vista que a Sociedade Brasileira de Cardiologia ${ }^{4}$ considera esse como tempo mínimo de internação, a fim de evitar possíveis complicações.

Em relação ao desfecho clínico, $89,7 \%$ foram de alta, isto é, houve transferência intra hospitalar; destaca-se que não houve casos de transferência externa.

\section{DISCUSSÃO}

Diante dos dados sociodemográficos desse estudo, constatase que houve predominância do sexo masculino, corroborando com estudos ${ }^{9-10}$, nos quais destacaram que os homens têm dificuldade em reconhecer suas necessidades de saúde e acabam negligenciando, buscando os serviços hospitalares apenas em casos agudizados. Nesse contexto, mostra-se necessário buscar alternativas de promoção de saúde e prevenção de doença para os homens, a fim de garantir um cuidado integral.

Em estudos nos estados do Sudeste e do Centro-oeste brasileiro ${ }^{9-11}$ apontam que o predomínio da busca pelo serviço de saúde é de pessoas com idade acima de 60 anos, tendo em vista que é a faixa etária que mais cresce em proporção populacional, em consequência ao aumento da expectativa de vida. Esses fatos, confirmam que, com o aumento do envelhecimento populacional, consequentemente acrescem também a frequência de usuários mais idosos com agravos à saúde que exigem tratamento em UTI, uma vez que a incidência de doenças crônicas degenerativas, dentre eles as doenças cardiovasculares, que aumentam com o avançar da idade. 
Assim sendo, pode-se justificar que, com o aumentar da idade, tende-se a alterar o metabolismo orgânico e em especial as artérias, as quais perdem elasticidade e se enrijecem. Esta ocorrência, pode tornar mais suscetível a lesões, assim como facilitam a formação de trombos, no qual pode obstruir o vaso sanguíneo e contribuir para o desenvolvimento do IAM. ${ }^{12}$

Em relação a procedência de internação na UTI, estudos realizados na região sul do Brasil $^{13-14}$ contradizem os achados nesse estudo, sendo que ambos apresentam prevalência do centro cirúrgico, por estarem relacionados a maior incidência de intervenções cirúrgicas emergenciais nas instituições.

Entretanto, no presente estudo foi evidenciado que a maioria dos pacientes são admitidos advindos do serviço de urgência e emergência. Visando a magnitude do IAM e seus fatores de risco, os usuários que são atendidos nas primeiras horas do aparecimento dos sintomas, são favorecidos por uma assistência precoce, reduzindo

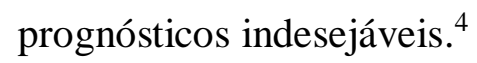

$\mathrm{Na}$ avaliação inicial, menos de $20 \%$ das vítimas de IAM apresentam alterações cardiovasculares, o que pode colocar o usuário em maior risco de desenvolver complicações, portanto, após o usuário adentrar ao serviço de urgência e emergência, faz-se necessário o encaminhamento do mesmo à UTI, tendo em vista a necessidade de monitorização eletrocardiográfica. Isto se deve pelo motivo da causa mais comum de morte pós IAM serem as arritmias, além do cuidado intensivo e terapêutica medicamentosa, com tempo mínimo de internação de, pelo menos, 72 horas. ${ }^{15-16,4}$

No que se refere ao tempo de permanência, em um estudo realizado no Rio Grande do $\mathrm{Sul}^{17}$ demonstrou dado similar ao desse estudo, com média entre 48 a 96 horas. Reflete-se que trata de um ambiente hospitalar com cuidados intensivos, na busca de estabilizar o usuário com o menor tempo possível, pois a demora no período de permanência na UTI associase ao mau prognóstico na recuperação do usuário, bem como ônus financeiro institucional.

Em um estudo realizado em uma cidade do interior de um estado no centrooeste brasileiro $^{10}$, ao analisar dados de três UTIs adulto distintas, foram constatados que, em todas houve predomínio dos motivos de internação de doenças cardiovasculares, com $45 \%$, sendo que o motivo de maior frequência foram o IAM, com 7\%; destes, ao analisar os casos de desfecho clínico, os dados entram em consonância com os do presente estudo, apresentando maior predominância dos casos de alta da UTI, os quais foram encaminhados para outras unidades da instituição. 


\section{CONCLUSÃO}

Mediante as características das vítimas de IAM admitidas, pode-se constatar que foram predominantes usuários do sexo masculino, com idade entre 65 e 74 anos e procedência dos serviços de urgência e emergência. Quanto ao desfecho clínico houve destaque para a alta hospitalar e o tempo de permanência na unidade foram de até 72 horas.

Evidencia-se que o conhecimento dos dados sociodemográficos e clínicos das vítimas atendidas pode favorecer no planejamento e efetivação da assistência, implicando melhorias na qualidade da mesma, podendo centrar o atendimento nas especificidades dos usuários, reestabelecendo uma medida preventiva, buscando uma evolução positiva no estado clínico geral e evitando um novo evento obstrutivo. Considera-se como limitação do estudo o fato de tais características refletirem apenas a realidade local.

AGRADECIMENTOS: Aos nossos colegas residentes e graduandos do Grupo de Estudos e Pesquisa de Enfermagem em Cardiologia (GEPEN Cardio) e em especial aos discentes Wanmar de Souza Oliveira e Anna Beatriz Ketsia Ribeiro Moreira Bazzano, que nos auxiliaram na tradução dos resumos deste presente estudo.

Fontes de financiamento: Fundação de Amparo à Pesquisa do Estado de Mato Grosso (FAPEMAT).
Vinculação do manuscrito: trabalho de conclusão de curso apresentado a Universidade Federal de Mato GrossoCâmpus Rondonópolis.

\section{REFERÊNCIAS}

1. Magalhães CC, Serrano Junior CV, Consolim- Colombo FM, Nobre F, Fonseca FAH, Ferreira JFM. Tratado de cardiologia SOCESP. $3^{\circ}$ ed. São Paulo (SP): Manole; 2015.

2. Mertins SM, Kolankiewiz ACB, Rosanelli CLSP, Loro MM, Poli G, Winkelman ER, et al. Prevalencia de factores de riesgo en pacientes con infarto agudo de miocárdio. Av Enferm [internet]. 2016; 34(1):30-8. Disponible: doi: http://dx.doi.org/10.15446/av.enferm.v34n 1.37125 .

3. Aehlert, B. Síndromes Coronarianas Agudas. In: Aehlert, B (ed.) ACLS:

Suporte avançado de vida em cardiologia: emergência em cardiologia. $4^{\circ}$ ed. Rio de Janeiro (RJ): Elservier; 2013. p. 211-75. 4. Piegas LS, Timerman A, Feitosa GS, Nicolau JC, Mattos LAP, et al. V Diretriz da Sociedade Brasileira de Cardiologia sobre Tratamento do Infarto agudo do Miocárdio com Supradesnível do Segmento ST. Arq Bras Cardiol. 2015 [acesso em 02 de dez 2017]; 2(105). Disponível em: http://publicacoes.cardiol.br/2014/diretrize s/2015/02_TRATAMENTO\%20DO\%20I AM\%20COM\%20SUPRADESNIVEL\%2 0DO\%20SEGMENTO\%20ST.pdf.

5. Brasil. Ministério da Saúde. Resolução $\mathrm{n}^{\circ}$ 137, de 8 de fevereiro 2017. Dispõe sobre os requisitos mínimos para funcionamento das Unidades de Terapia Intensiva e dá outras providências. Diário Oficial da União 09 fev 2017; Seção 1. p. 44.

6. Silva RB, Castro CM, Iser BPM, Castilho LJC. Perfil dos pacientes com síndromes coronarianas agudas em um hospital da Região Sul do Brasil. Rev Soc Bras Clin Med [periódico online]. 2016 
[acesso em 06 mai 2018]; 14(1):33-7.

Disponível em:

http://pesquisa.bvsalud.org/portal/resource/ pt/biblio-18.

7. Brasil. Ministério da Saúde.

Departamento de Informática do Sistema

Único de Saúde (DATASUS). Informações

de Saúde [publicação online]. 2018 [acesso em 22 jun 2018]; Disponível em:

http://datasus.saude.gov.br/informacoes-

de-saude.

8. Brasil. Ministério da Saúde. Resolução

no 466, de 12 de dezembro de 2012.

Conselho Nacional de Saúde. Brasília,

2012 [acesso em 11 de out 2017].

Disponível em:

http://conselho.saude.gov.br/resolucoes/20

12/Reso466.pdf .

9. Soriano KS, Pires DBP, Melo LS,

Chaves SDDRB, Salviano MEM, Tannure

MC. Perfil de pacientes vítimas de infarto

agudo do miocárdio internados em uma

unidade coronariana de Belo Horizonte.

Enfermagem Revista [periódico online].

2016 [acesso em 30 abr 2018]; 1(19):21-9.

Disponível em:

http://periodicos.pucminas.br/index.php/en

fermagemrevista/article/view/11632.

10. Castro RR, Barbosa NB, Alves T,

Najberg E. Perfil das Internações em

Unidades de Terapia Intensiva Adulto na

Cidade de Anápolis - Goiás - 2012. Revista

de Gestão em Sistemas de Saúde. 2016;

2(5):115-24. Disponível em: doi:

10.5585/rgss.v5i2.243.

11. Dordetto PR, Pinto GC, Rosa TCSC.

Pacientes submetidos à cirurgia cardíaca:

caracterização sociodemográfica, perfil

clínico-epidemiológico e complicações.

Rev Fac Ciênc Méd Sorocaba. 2016;

18(3):144-9. Disponível em: doi:

10.5327/Z1984-4840201625868.

12. Lima AEF, Lima LD, Sandes TKS,

Oliveira Neto JF, Silva KMM, Pereira RB.

Perfil na mortalidade por infarto agudo do

miocárdio por idade e sexo no município de Paulo Afonso no estado da Bahia.

Revista Rios Saúde [periódico online].

2018 [acesso em 22 jun 2018]; 3(1):26-37.

Disponível em:

https://www.fasete.edu.br/revistariossaude/

13. Rodriguez AH, Bub MBC, Perão OF,

Zandonadi G, Rodriguez MJH.

Epidemiological characteristics and causes of deaths in hospitalized patients under intensive care. Rev Bras Enferm [Internet]. 2016; 69(2):210-4. Available from: doi: http://dx.doi.org/10.1590/0034-

7167.2016690204i.

14. Nogueira L de S, Sousa RMC, Padilha KG, Koike KM. Clinical characteristics and severity of patients admitted to public and private icus. Texto Contexto Enferm. 2012; 21(1):59-67. Available from: doi: http://dx.doi.org/10.1590/S010407072012000100007.

15. Medeiros TLF, Andrade PCNS, Davim RMB, Santos NMG. Mortality by an acute myocardial infarction. Rev Enferm UFPE [internet]. 2018; 12(2):565-72. Available from: doi: http://doi.org/10.5205/19818963-v12i2a230729p565-572-2018.

16. Ouchi JD, Teixeira C, Góes Ribeiro CA, Oliveira CC. Tempo de Chegada do Paciente Infartado na Unidade de Terapia Intensiva: a Importância do Rápido Atendimento. Ensaios Cienc, Cienc Biol Agrar. Saúde. 2017; 21(2):92-7.

Disponível em: doi: 10.17921/14156938.2017v21n2p92-97.

17. Silveira CR, Santos MBK, Moraes MAP, Souza EN. Desfechos clínicos de pacientes submetidos à cirurgia cardíaca em um hospital do noroeste do Rio Grande do Sul. Rev Enferm UFSM. 2016; 6(1):102-11. Disponível em: doi: $10.5902 / 2179769216467$

RECEBIDO: 28/05/2019

APROVADO: 01/10/2019

PUBLICADO: 12/2019 